\title{
USE SATELLITE IMAGES AND IMPROVE THE ACCURACY OF HYPERSPECTRAL IMAGE WITH THE CLASSIFICATION
}

\author{
Peyman Javadi a ,* \\ ${ }^{\text {a }}$ Ms Student in Geomatic, Department of Surveying Eng, Islamic Azad University, Taft, Iran - peymanjavadi_ir@yahoo.com
}

KEY WORDS: Accuracy Improvement, Hyperspectral, Minimum Noise, Classification, Subpixel.

\begin{abstract}
:
The best technique to extract information from remotely sensed image is classification. The problem of traditional classification methods is that each pixel is assigned to a single class by presuming all pixels within the image. Mixed pixel classification or spectral unmixing, is a process that extracts the proportions of the pure components of each mixed pixel. This approach is called spectral unmixing. Hyper spectral images have higher spectral resolution than multispectral images. In this paper, pixel-based classification methods such as the spectral angle mapper, maximum likelihood classification and subpixel classification method (linear spectral unmixing) were implemented on the AVIRIS hyper spectral images. Then, pixel-based and subpixel based classification algorithms were compared. Also, the capabilities and advantages of spectral linear unmixing method were investigated. The spectral unmixing method that implemented here is an effective technique for classifying a hyperspectral image giving the classification accuracy about $89 \%$. The results of classification when applying on the original images are not good because some of the hyperspectral image bands are subject to absorption and they contain only little signal. So it is necessary to prepare the data at the beginning of the process. The bands can be stored according to their variance. In bands with a high variance, we can distinguish the features from each other in a better mode in order to increase the accuracy of classification. Also, applying the MNF transformation on the hyperspectral images increase the individual classes accuracy of pixel based classification methods as well as unmixing method about 20 percent and 9 percent respectively.
\end{abstract}

\section{1) Introduction}

By remote sensing we can view the earth, through windows of the electromagnetic spectrum that would not be humanly possible. Remote sensing includes both data acquisition and data interpretation. The image analysis part can be added to the data acquisition system to model the entire RS system. The analysis part is a key component of RS system, because we can change the data to information via it. The most important technique to extract information, from remotely sensed images, is classification. .The limitation of traditional classification methods is that each pixel is assigned to in a single class by presuming all pixels within the image are pure. The aim of this research can be explained by looking at the problems of mixed pixels in classification. These aims are: processing of mixed pixels, the primary aim of this research is evolution and processing of mixed pixels to improvement accuracy of the classification results and classification of mixed pixels, the seconding aims of this research is to provide knowledge about the decomposition of mixed pixels (spectral unmixing) on the hyperspectral image. New image processing tools and techniques, such as spectral unmixing, can be investigated through introducing new sensors with hyperspectral capabilities. Then, next section introduces the preparation of data for classification methods. Section 3 discusses the experimental data and evaluation and compares works that are performed on the hyperspectral images. Section 4, concludes the research.

\section{2) Noise and its Removing from the Image Data}

Hyperspectral image bands are often highly correlated and among them some of absorption bands contain little signal but noise. Analysis of the original spectral bands is inefficient and creates poor results. The MNF transformation was applied on the image to separate the image noise and extract the signal from the original bands. For transformation, noise statistics of the data were conjectured. Estimating the pixel's noise was carried out by a shift difference on a specific homogenous area of the image by distinguishing adjacent pixels to each pixel and averaging the findings. The MNF transform is essentially two principal components transformations. The first transformation is based on an estimated noise covariance matrix. This first step results in transformed data in which the noise has unit variance and no band to band correlations. The second step is a standard principal components transformation. The MNF transform was carried out the AVIRIS images, resulting an output of 195 components. These components were sorted regarding to their variance. Almost $99 \%$ of total variance is presented in the 50 first components.

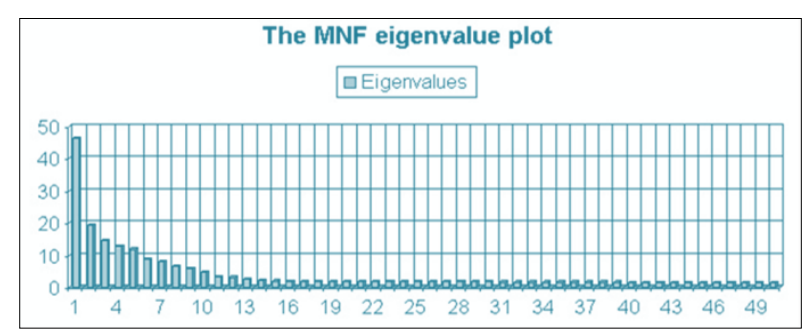

Figure 1. The MNF eigenvalue plot.

Shows the cutoff number which was determined by checking the MNF eigenvalue plot. Transforming MNF, the data space can be divided into two parts: one part associated with large eigenvalues and corresponding eigenimages, and the other part having near-unity eigenvalues are noise dominated images. Visual assessment of images reveals that the bands numbered greater than 150 consist of data dominated by noise. Figure 2 shows the first three bands (1,2 and 3) of MNF images which have large eigenvalues and corresponding eigenimages. Also, Figure 3 shows the last three bands (193, 194 and 195) of MNF images which are images dominated by noise. 


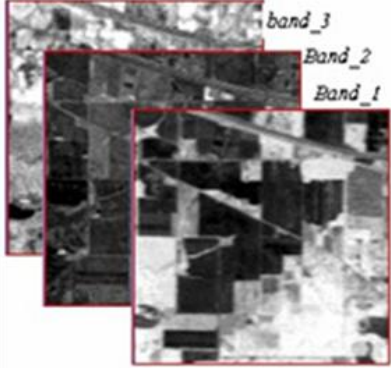

Figure 2 (A). MNF (1, 2 and 3) bands

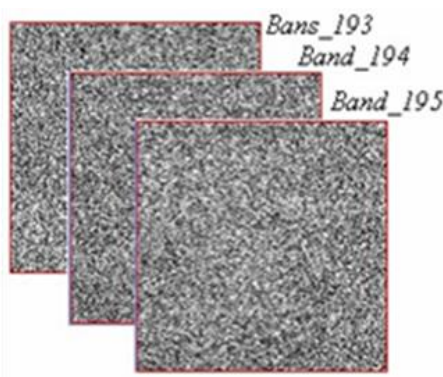

Figure 3 (B). MNF (193, 194 and 195) bands

\section{3) Excrements}

In this research, pixel-based classification methods such as the spectral angle mapper, maximum likelihood classification and subpixel classification method (linear spectral unmixing) were implemented on the AVIRIS hyperspectral images. Then in order to process the mixed pixels in classification results, pixel-based and subpixel based classification algorithms were compared.

\section{1) Pixel-Based Classification Methods}

The probability theory to the classification task is applied by MLC method and all of the probabilities of classes for each pixel and assigning that pixel to the class with the highest probability value are computed by it. Also, the SAM considers image spectra as vectors in n-dimensional spectral space. Each spectrum defines a point in spectral space. The angle between a pair of vectors, i.e., reference spectrum and image spectrum, is a measure of the similarity of the spectra; smaller spectral angles indicate greater similarity. The differences in brightness average between spectra are important for this method, because these change the length of the spectral vector, but not its orientation. These methods was applies upon MNF images as well as on the original bands. In these procedures, 3, 5, 10, 20, 50, 87, 120, 155,175 and 195 bands were used for classification.

\section{2) Sub-Pixel Classification Method}

The main problem of traditional image classification procedures in classification of mixed pixel is that a single class belongs to each pixel by presuming all pixels within the image are pure. The process of mixed pixel classification tries to extract the features of the pure components of each mixed pixel. There are different models to cope with the mixed pixel problem. The nonlinear mixture model considers both the pixel of interest and the neighboring pixels i.e. each photon that reaches the sensor has interacted with multiple scattering between the different class types. The linear mixture model that assumes each pixel is modeled as a linear combination of a number of pure materials or endmembers [3]. The linear mixture model is known as spectral unmixing. It is a method by which the user can ascertain information on a subpixel level and study decomposition of mixed pixels. The main idea of linear mixture model is that, each photon that reaches the sensor has interacted with just one class.

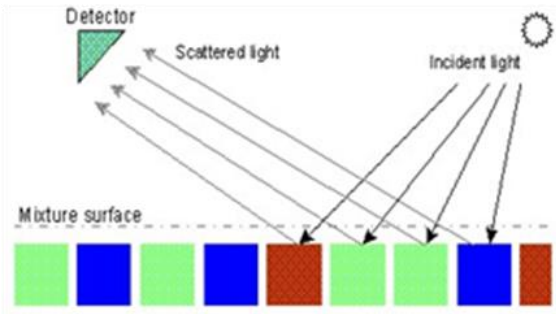

Figure 4. The linear mixture model

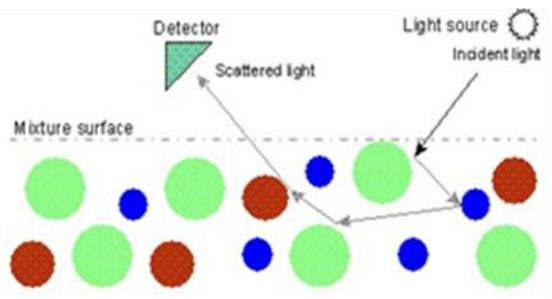

Figure 5. Non-linear mixture model

One of the limitations of non-linear spectral unmixing is that it is necessary to know the exact imaging geometry (i.e. incident and viewing angle) under which each image is captured. For accurate results one has to model the reflectivity function of each material, i.e. how much light is reflected in each direction in each wavelength. The equations relating to imaging geometry, reflectance is nonlinear. Also many surface material mix in non-linear fashions but linear unmixing method, while at best an approximation, appear to work well in many application (Boardman and Kruse, 1994). The linear mixture model which is used in this paper mixture can be mathematically described as a set of linear vector-matrix equation,

$$
D N_{i}=\sum_{j=1}^{n}\left(E_{i j} \cdot F_{j}\right)+\varepsilon_{i} \quad \text { OR } \quad D N=E F+\varepsilon
$$

Where

- $\mathrm{i}=1, \ldots, \mathrm{m}$ (number of bands).

- $j=1, \ldots, n$ number of endmembers or classes).

- $\mathrm{DN}_{\mathrm{i}}$ is the $\mathrm{i} \times 1$ multi or hyperspectral vector at each pixel.

- $E_{\mathrm{ij}}$ is the $\mathrm{j} \times 1$ endmember spectrum matrix and it is the weighting fraction of each end member. Each column in $\mathrm{E}_{\mathrm{ij}}$ matrix is the spectrum of one endmember.

- $F_{j}$ is the $\mathrm{j} \times 1$ fraction vector of each endmember for pixel.

- $\varepsilon_{\mathrm{i}}$ is the error term of mathematical model

$$
D N=\left[\begin{array}{c}
D N_{1} \\
D N_{2} \\
\ldots \\
D N_{m}
\end{array}\right] ; E=\left[\begin{array}{ccc}
E_{11} & E_{12} & E_{1 n} \\
E_{21} & E_{22} & E_{2 n} \\
& & \\
E_{m 1} & E_{m 2} & E_{m n}
\end{array}\right] ; F=\left[\begin{array}{c}
F_{1} \\
F_{2} \\
\ldots \\
F_{m}
\end{array}\right] ; \varepsilon=\left[\begin{array}{c}
\varepsilon_{1} \\
\varepsilon_{2} \\
\ldots \\
\varepsilon_{m}
\end{array}\right]
$$

Solving of the equation 3.1 is unconstrained unmixing. Maybe the inferred fractions have negative values and are not constrained to sum to unity. This unit to sum constraint is added to the system of equations in the unmixing process. Applying 
the condition that all the resulting fractions must sum to unity is referred to partially constrain unmixing. However, fraction values which are negative or greater than one are still possible. Fully constrained unmixing implies an additional condition in that all determined endmember fractions must be between 0 and 1. The final results from unmixing algorithm depend on to type and number of input endmembers. Therefore, any changes applied to the reference endmembers lead to changes on the fraction map results.

In order to solve the linear unmixing problem; the sum of the coefficients should equals one, because ensure the whole pixel area is represented in the model and also each of the fraction coefficients be nonnegative to avoid negative subpixel areas. The constraint equation, can model the first requirement, for the second requirement, the coefficients need to be constrained by

inequality: $F_{n} \geq 0, \sum_{n=1}^{N} F_{n}=1$

Together, the mixing equations and the constraints describe a model that must be solved for each pixel which should be decomposed, i.e. given $\mathrm{DN}$ and $\mathrm{E}$, we have to determine $\mathrm{F}$ and $\varepsilon$ in equation (1).

\section{4) Experiments with Pixel-Based Classification Methods}

The image of Figure 6, which is a portion of the Airborne Visible/Infrared Imaging Spectrometer (AVIRIS) of hyperspectral data taken over an agricultural area of California, USA in 1994 was applied in this paper. This data has 220 spectral bands about $10 \mathrm{~nm}$ apart in the spectral region from 0.4 to $2.45 \mu \mathrm{m}$ with a spatial resolution of $20 \mathrm{~m}$. The image has a pixel of 145 rows by 145 columns. Figure 7. Shows the corresponding ground truth map. It includes of 12 classes.

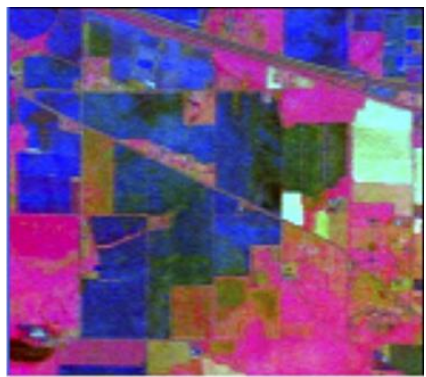

Figure 6. RGB (31,19 and 9) MNF of image

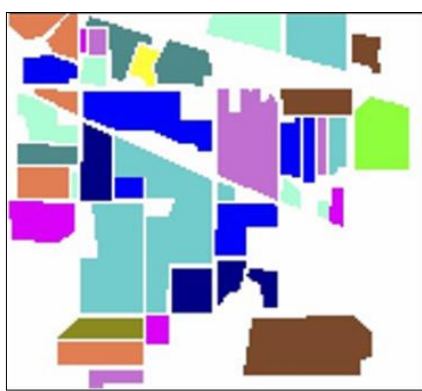

Figure 7: The ground truth map

\section{1) Maximum Likelihood Classification (MLC)}

The MLC method was applied upon MNF images as well as on the original bands. This image has 220 spectral bands which 25 bands are noisy. Therefore 195 bands have been used in this research. In this procedure, 3, 5, 10, 20, 50, 87, 120, 155, 175 and 195 bands was used for classification. Table (1) shows
Overall Accuracy (OA) and Kappa coefficient $(\mathrm{K})$ for this method.

\begin{tabular}{|c|c|c|c|c|c|c|c|}
\hline \multirow{2}{*}{\multicolumn{2}{|c|}{ Type of images }} & \multicolumn{2}{|c|}{ MLC } & \multirow{2}{*}{\multicolumn{2}{|c|}{ Type of images }} & \multicolumn{2}{|c|}{ MLC } \\
\hline & & \multirow[t]{2}{*}{ org } & \multirow{2}{*}{$\mathrm{mnf}$} & & & org & $\mathrm{mnf}$ \\
\hline N.B & & & & N.B & & & \\
\hline \multirow{2}{*}{3} & $\mathrm{OA}(\%)$ & 22.98 & 57.93 & \multirow{2}{*}{87} & OA (\%) & 65.89 & 77.92 \\
\hline & $\mathrm{K}(\%)$ & 15.98 & 53.58 & & $\mathrm{~K}(\%)$ & 61.68 & 75.17 \\
\hline \multirow[t]{2}{*}{5} & $\mathrm{OA}(\%)$ & 31.11 & 61.33 & \multirow{2}{*}{120} & $\mathrm{OA}(\%)$ & 79.54 & 81.04 \\
\hline & $\bar{K}(\%)$ & 24.38 & 57.29 & & $\overline{\mathrm{K}(\%)}$ & 76.84 & 78.62 \\
\hline \multirow{2}{*}{10} & $\mathrm{OA}(\%)$ & 36.29 & 64.88 & \multirow{2}{*}{155} & $\mathrm{OA}(\%)$ & 13.83 & 55.41 \\
\hline & $\mathrm{K}(\%)$ & 29.66 & 61.01 & & $\mathrm{~K}(\%)$ & 00.00 & 51.25 \\
\hline \multirow[t]{2}{*}{20} & $\mathrm{OA}(\%)$ & 54.78 & 69.07 & \multirow[t]{2}{*}{175} & $\mathrm{OA}(\%)$ & - & 43.01 \\
\hline & $\mathrm{K}(\%)$ & 49.73 & 65.51 & & $\mathrm{~K}(\%)$ & - & 38.92 \\
\hline & $\overline{\mathrm{OA}}(\%)$ & 59.91 & 73.71 & \multirow{2}{*}{195} & $\overline{\mathrm{OA}(\%)}$ & - & 41.84 \\
\hline & $\mathrm{K}(\%)$ & 55.21 & 70.52 & & $\mathrm{~K}(\%)$ & - & 37.5 \\
\hline
\end{tabular}

Table 1. The Overall Accuracy (OA) and Kappa coefficient (K) for MLC classification

In the above Table N.B refers to the number of bands that were used for classification. Key words org, mnf refers to the original and minimum noise fraction bands, respectively.

\section{2) Spectral Angle Mapper}

The Spectral Angle Mapper applies upon MNF images as well as original bands. In this procedure $3,5,10,20,50,87,120$, 155, 175 and 195 bands were used for classification. Table (2) shows Overall Accuracy (OA) and Kappa coefficient (K) for this method.

\begin{tabular}{|c|c|c|c|c|c|c|c|}
\hline \multirow{2}{*}{\multicolumn{2}{|c|}{ Method }} & \multicolumn{2}{|c|}{ SAM } & \multirow{2}{*}{\multicolumn{2}{|c|}{ Method }} & \multicolumn{2}{|c|}{ SAM } \\
\hline & & \multirow[t]{2}{*}{ org } & \multirow[t]{2}{*}{$\mathrm{mnf}$} & & & \multirow[t]{2}{*}{ org } & \multirow[t]{2}{*}{$\mathrm{mnf}$} \\
\hline $\mathrm{VR}$ & & & & N.B & & & \\
\hline \multirow{2}{*}{3} & & 6.23 & 46.00 & \multirow{2}{*}{87} & $\mathrm{OA}$ & 42.79 & 71.02 \\
\hline & & 1.61 & 40.41 & & ) & 37.07 & 67.39 \\
\hline & & 14.96 & 54.56 & \multirow{2}{*}{120} & & 44.84 & 74.86 \\
\hline & $\mathrm{K}(\%)$ & 9.16 & 49.58 & & & 39.05 & 71.92 \\
\hline \multirow{2}{*}{10} & & 24.11 & 63.09 & \multirow{2}{*}{155} & OA (\%) & 45.86 & 70.45 \\
\hline & & 18.03 & 58.74 & & & 39.98 & 66.74 \\
\hline \multirow{2}{*}{20} & & 28.24 & 66.37 & \multirow{2}{*}{175} & & 45.97 & 72.66 \\
\hline & & 22.35 & 62.27 & & $\mathrm{~K}(\%)$ & 40.10 & 69.33 \\
\hline \multirow{2}{*}{50} & $\mathrm{OA}(\%)$ & 42.28 & 68.97 & \multirow{2}{*}{195} & $\mathrm{OA}(\%)$ & 45.94 & 73.99 \\
\hline & $\mathrm{K}(\%)$ & 36.56 & 65.07 & & $\mathrm{~K}(\%)$ & 40.05 & 70.8 \\
\hline
\end{tabular}

Table 2. The Overall Accuracy (OA) and Kappa coefficient (K) for SAM classification

The classification accuracy can be improved by Increasing the number of bands in every two types of data. Analysis of all of the original spectral bands tends to create poor results. The classification accuracy of MNP bands are higher than the original images, because the hyperspectral image bands are often highly correlated and among them some of absorption bands contain little signal but more noise content. Using the MNF transformation on the original bands, removes the correlation between bands, separates the noise and improves the 
classification accuracy. Therefore the classification accuracy is improved. Visual and eigenvalue assessment of MNF of AVIRIS data shows that the bands numbered greater than 120 consists of data which dominated by noise. So increasing the number of bands greater than 120 , decreases accuracy of the classification results. Comparing the classification results of MLC classification and SAM shows that the MLC has higher accuracy than SAM classification.

\section{3) Subpixel Classification (Linear Spectral Unmixing)}

The foundation of linear unmixing is that the most of pixels are mixtures of objects. Once all the endmembers are found in an image, all the remaining pixels are considered to be linear combinations of these endmember pixels. This method was applied upon MNF images as well as original bands. In this procedure, 20, 50, 87, 120,155, 175 and 195 number of bands were used for classification. The eight fraction images resulted of LSU have been showed in the Figure 8.
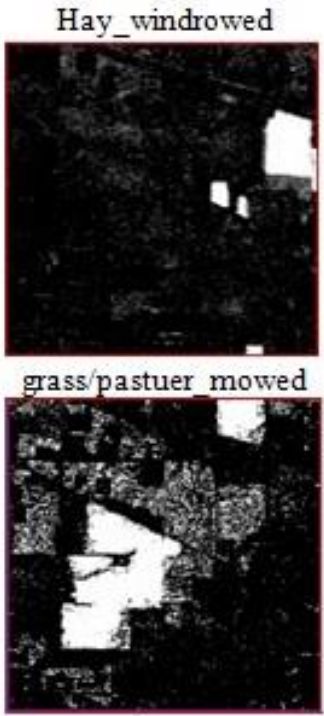

Oatas

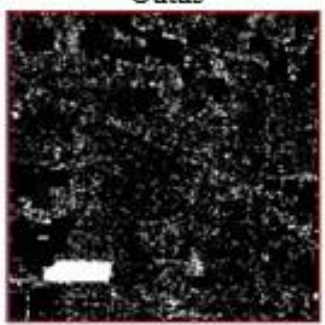

soybeans notill

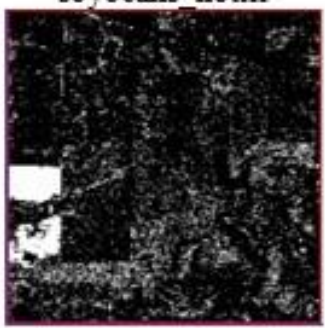

Figure 8. The fraction images of classes from the LS unmixing which is performed on the MNF bands.

Although the fraction images represent the results of subpixel classification and they do not show directly the distribution of classified areas. However, by choosing the class with the largest pixel value in the fraction images for output, one can generate a class map that is a pixel-based representation of classification.

4.3.1) Evaluation of Linear Spectral Unmixing: After each classification its results must be evaluated and their accuracy must be assessed. According to the results type (thematic map, fraction map), an adequate strategy for accuracy assessment can be chosen. Finally to reveal the accuracy of the results, some parameters, tables and maps will be calculated and generated. Methods of accuracy assessment for traditional pixel-based classification are not fully suitable for subpixel classification. Because, our training data and ground truth are pixel-based and we couldn't find any sub-pixel based method for accuracy assessment

There are no evaluation methods for subpixel classification and the traditional accuracy assessment procedures can't be applied for subpixel accuracy assessment. or subpixel accuracy assessment, we have to use fraction maps as the main results of the linear unmixing classification. In the first step parameter is necessary to express the matching rate of the results with the ground truth. So we introduce the Correctness Coefficient (CC) method. Also, several procedures for subpixel accuracy assessment were introduced. A binary map from the ground truth is generated for each class to calculate of the Correctness Coefficient (CC) parameter. The numbers of binary images are equal to the number of classes or endmembers. In this research, 12 binary images are produced. The gray value of pixels in a binary image is 0 or 1 . Number 1 indicates that total of this pixel belongs to the corresponding class of binary image and number 0 indicates that this pixel does not belong to corresponding class. In contrast, the results of spectral unmixing method are fraction maps in which pixel values are numbers between 0 to 1 . Pixel to pixel multiplying of ground truth binary image and corresponding fraction map is an image with pixel values between zero and one.

For CC calculation, the sum of the pixel values in the above produced image is divided to the number of pixels in the land use for each fraction map. The result is a number between 0 and 1 which is named CC for linear spectral unmixing results. All pixels in land use and fraction map are the same labeled and the unmixing correctness is $100 \%$, if CC equals 1 . If CC is equal to zero then it means that no pixel in land use and fraction map has the same label and the unmixing correctness is $0 \%$. Conceptually, this parameter is expressing the matching rate of the results of unmixing the ground truth. In this research CC calculated for each class. Total CC is calculated for linear unmixing, too. Result of this operation is shown in Table 3.

\begin{tabular}{|c|c|}
\hline Classes & Correctness Coefficient \\
\hline Alfalfa & 0.9641 \\
\hline Corn & 0.8577 \\
\hline Corn_min & 0.8211 \\
\hline Corn_notill & 0.8596 \\
\hline Grass_pasture & 0.7071 \\
\hline Grass_pasture_mov & 0.8546 \\
\hline Grass_Trees & 0.9447 \\
\hline Hay_windrowed & 0.9271 \\
\hline Oatas & 0.9838 \\
\hline Soybeans_clean & 0.9122 \\
\hline Soybeans_notill & 0.8237 \\
\hline Wood & 0.7623 \\
\hline
\end{tabular}

Table 3. The calculation Correctness Coefficient (CC) for linear unmixing on the MNF images 
The mean of the correctness coefficients for linear unmixing is 0.8459 that indicates the result of unmixing, i.e., fraction maps are fitted with land use about $85 \%$. This coefficient is similar to overall accuracy for pixel-based classification procedures.

4.3.2) Accuracy Assessment of Linear Spectral Unmixing Results: Accuracy assessment indices in traditional pixel-based classification methods, i.e., overall accuracy and kappa coefficient, are not suitable for subpixel classification accuracy assessment. Training data and ground truth are pixel based and we don't have any subpixel based method of accuracy assessment. In error matrix of traditional classification methods, commission error defines the percentage of those pixels that have been labeled as a particular class but in ground truth are in a different category. Omission error also defines the percentage of pixels from a particular class which have been labeled as the other classes. So omission and commission errors for each class can be calculated by using subpixel classification results.

For this purpose, first the ground truth of the image converted to binary images. The numbers of binary images and the number of classes or endmembers are the same. In this research, 12 binary images are produced. The gray value of pixels in a binary image is 0 or 1 . In contrast, the results of spectral unmixing method are fraction maps in which pixel values are numbers among 0 to 1 . Pixel to pixel differencing of ground truth binary image and corresponding fraction map is an image in which the pixel values are negative, zero or positive. A zero pixel value indicates that this pixel was correctly classified, i.e., $100 \%$. Negative pixel value indicates the incorrectly classified percent of that pixel and belongs to other classes in the ground truth. This error is equivalent with the commission error in the traditional pixel-based classification. Similarly, positive pixel values indicates the percent of pixel that does not classified correctly but in ground truth belongs to this class. This error is equivalent with omission error in traditional pixel-based classification. The Table (4) shows result of the calculation.

\begin{tabular}{|c|c|c|c|c|}
\hline Classes & Comm & Omm & User & Prod \\
& & & $\begin{array}{c}\text { Accuracy } \\
(\%)\end{array}$ & $\begin{array}{c}\text { Accuracy } \\
(\%)\end{array}$ \\
\hline Hay_windrowed & 11.3354 & 7.2874 & 88.6646 & 92.7126 \\
\hline Corn_min & 34.9005 & 12.7987 & 65.0995 & 87.2013 \\
\hline Corn_notill & 10.6819 & 15.6105 & 89.3181 & 84.3895 \\
\hline Corn & 32.9771 & 19.6302 & 67.0229 & 80.3698 \\
\hline Grass_pasture_moved & 20.0045 & 17.7140 & 79.9955 & 82.2860 \\
\hline Soybeans_clean & 41.1789 & 9.7694 & 58.8211 & 90.2306 \\
\hline Grass_Trees & 33.5519 & 5.5348 & 66.4481 & 94.4652 \\
\hline Grass_pasture & 11.2371 & 23.1949 & 88.7629 & 76.8051 \\
\hline Soybeans_notill & 35.1226 & 22.7795 & 64.8774 & 77.2205 \\
\hline Wood & 9.9695 & 3.7110 & 90.0305 & 96.2890 \\
\hline Alfalfa & 9.9695 & 3.7110 & 90.0305 & 96.2890 \\
\hline Oatas & 16.0624 & 1.6241 & 83.9376 & 98.3759 \\
\hline
\end{tabular}

Table 4. Estimation of error in linear spectral unmixing for original images (120 bands)

According to the findings of original images, the average producers accuracy is $88.05 \%$ and user's accuracy is $77.75 \%$ for classes. These accuracy parameters on the MNF images are shown in Table (5).

\begin{tabular}{|c|c|c|c|c|}
\hline Classes & Comm & Omm & User & Prod \\
Error (\%) & Error (\%) & $\begin{array}{c}\text { Accuracy } \\
(\%)\end{array}$ & $\begin{array}{c}\text { Accuracy } \\
(\%)\end{array}$ \\
\hline Hay_windrowed & 11.8156 & 7.2874 & 88.1844 & 92.7126 \\
\hline Corn_min & 18.2697 & 12.1381 & 81.7303 & 87.8619 \\
\hline Corn_notill & 3.8830 & 12.8569 & 96.1170 & 87.1431 \\
\hline Corn & 30.4930 & 13.4164 & 69.5070 & 86.5836 \\
\hline Grass_pasture_moved & 3.2760 & 12.7402 & 96.7240 & 87.2598 \\
\hline Soybeans_clean & 17.7028 & 8.2870 & 82.2972 & 91.7130 \\
\hline Grass_Trees & 33.5519 & 5.5348 & 66.4481 & 94.4652 \\
\hline Grass_pasture & 2.8436 & 24.4573 & 97.1564 & 75.5427 \\
\hline Soybeans_notill & 26.2526 & 15.8734 & 73.7474 & 84.1266 \\
\hline Wood & 1.2772 & 22.3032 & 98.7228 & 77.6968 \\
\hline Alfalfa & 8.6625 & 3.5913 & 91.3375 & 96.4087 \\
\hline Oatas & 16.0624 & 1.6241 & 83.9376 & 98.3759 \\
\hline
\end{tabular}

Table 5. Estimation of error in linear spectral unmixing method for MNF images (120 bands)

Based in the results on the MNF images, it can be said that the average producers accuracy is $88.32 \%$ and user's accuracy is $85.49 \%$ for classes (see Table 5). It is clear, that applying MNF on the original images, users and producers accuracy is improved. The overall accuracy can be used to summarize the classification results. Table (6) shows the OA estimation for linear spectral unmixing classification results.

\begin{tabular}{|c|c|c|c|}
\hline Type of images & $\begin{array}{c}\text { On diag pixel } \\
\text { on } \\
\text { The Original } \\
\text { images (N) }\end{array}$ & $\begin{array}{c}\text { On diag pixel } \\
\text { on } \\
\text { The Mnf } \\
\text { images (N) }\end{array}$ & $\begin{array}{c}\text { Num pixel on } \\
\text { the Ground } \\
\text { truth (N) }\end{array}$ \\
\hline Hay_windrowed & 453.3647 & 453.3647 & 489 \\
\hline Corn_min & 679.2588 & 684.7680 & 834 \\
\hline Corn_notill & 1194.100 & 1232.600 & 1434 \\
\hline Corn & 488.4706 & 526.6235 & 614 \\
\hline Grass_pasture_moved & 2010.200 & 2131.300 & 2494 \\
\hline Soybeans_clean & 551.0157 & 560.1176 & 614 \\
\hline Grass_Trees & 661.7922 & 705.6549 & 747 \\
\hline Grass_pasture & 357.7216 & 351.4471 & 497 \\
\hline Soybeans_notill & 1194.100 & 1232.600 & 968 \\
\hline Wood & 968.0510 & 986.3961 & 1294 \\
\hline Alfalfa & 91.4745 & 91.5882 & 95 \\
\hline Oatas & 208.5569 & 208.5569 & 212 \\
\hline
\end{tabular}

Table 6. Estimation of overall accuracy (OA) for LSU classification (120 bands)

\section{5) Comparison between Accuracy of Sub-Pixel and Pixel- Based Classification}

This comparison was implemented between users and producers accuracy as well as overall accuracy of Subpixel and PixelBased Classification method for evolution of the decomposing of mixed pixel. Table (7) shows users and producer's accuracy for original images in the three classification method. 


\begin{tabular}{|c|c|c|c|c|c|c|c|}
\hline \multirow{2}{*}{ Method } & Org_Unmix & Org_MLC & Org_SAM \\
\cline { 2 - 8 } & $\begin{array}{c}\text { P.Acc } \\
(\%)\end{array}$ & $\begin{array}{c}\text { U.Acc } \\
(\%)\end{array}$ & $\begin{array}{c}\text { P.Acc } \\
(\%)\end{array}$ & $\begin{array}{c}\text { U.Acc } \\
(\%)\end{array}$ & $\begin{array}{c}\text { P.Acc } \\
(\%)\end{array}$ & $\begin{array}{c}\text { U.Acc } \\
(\%)\end{array}$ \\
\hline classes & \multicolumn{7}{|c|}{} \\
\hline Hay_windrowed & 92.72 & 88.67 & 92.84 & 76.82 & 99.39 & 55.48 \\
\hline Corn_min & 87.20 & 65.09 & 55.76 & 33.03 & 50.48 & 24.52 \\
\hline Corn_notill & 84.39 & 89.32 & 51.19 & 60.81 & 24.62 & 32.24 \\
\hline Corn & 80.37 & 67.02 & 34.87 & 41.36 & 14.43 & 24.7 \\
\hline $\begin{array}{c}\text { Grass_pasture__ } \\
\text { moved }\end{array}$ & 82.29 & 79.99 & 39.55 & 72.3 & 20.95 & 76.37 \\
\hline Soybeans_clean & 90.24 & 58.82 & 55.54 & 34.1 & 45.77 & 24.65 \\
\hline Grass_Trees & 94.47 & 66.45 & 77.11 & 79.89 & 48.19 & 71.43 \\
\hline Grass_pasture & 76.81 & 88.77 & 64.19 & 62.92 & 3.02 & 4.18 \\
\hline Soybeans_notill & 77.23 & 64.88 & 54.03 & 39.71 & 70.25 & 43.15 \\
\hline Wood & 76.13 & 88.69 & 76.43 & 62.24 & 88.1 & 74.85 \\
\hline Alfalfa & 96.29 & 90.04 & 96.84 & 36.65 & 100 & 39.09 \\
\hline Oatas & 98.38 & 83.94 & 96.23 & 64.15 & 93.4 & 82.85 \\
\hline
\end{tabular}

Table 7. The comparison users and producer's accuracy for original images (120 bands)

Also, the users and producer's accuracy for MNF images in the three classification method are shown in Table (8).

\begin{tabular}{|c|c|c|c|c|c|c|}
\hline \multirow{2}{*}{ Method } & \multicolumn{2}{|c|}{ Mnf_Unmix } & \multicolumn{2}{|c|}{ Mnf_MLC } & \multicolumn{2}{c|}{ Mnf_SAM } \\
\cline { 2 - 7 } & $\begin{array}{c}\text { P.Acc } \\
(\%)\end{array}$ & $\begin{array}{c}\text { U.Acc } \\
(\%)\end{array}$ & $\begin{array}{c}\text { P.Acc } \\
(\%)\end{array}$ & $\begin{array}{c}\text { U.Acc } \\
(\%)\end{array}$ & $\begin{array}{c}\text { P.Acc } \\
(\%)\end{array}$ & $\begin{array}{c}\text { U.A cc } \\
(\%)\end{array}$ \\
\hline Classes & \multicolumn{7}{|c|}{} \\
\hline Hay_windrowed & 92.71 & 88.18 & 90.39 & 90.59 & 100 & 75.7 \\
\hline Corn_min & 87.86 & 81.73 & 85.37 & 60.65 & 58.92 & 53.61 \\
\hline Corn_notill & 87.14 & 96.12 & 85.84 & 81.09 & 66.81 & 80.07 \\
\hline Corn & 86.58 & 69.51 & 58.26 & 65.62 & 30.25 & 90.48 \\
\hline $\begin{array}{c}\text { Grass_pasture_- } \\
\text { moved }\end{array}$ & 87.26 & 96.72 & 61.75 & 87.64 & 30.41 & 63.97 \\
\hline Soybeans_clean & 91.71 & 82.30 & 91.21 & 62.15 & 74.4 & 37.91 \\
\hline Grass_Trees & 88.59 & 90.27 & 62.25 & 80.17 & 95.31 & 76.29 \\
\hline Grass_pasture & 75.54 & 97.16 & 83.7 & 72.35 & 66.87 & 81.86 \\
\hline Soybeans_notilly & 84.13 & 73.75 & 75.62 & 71.48 & 63.13 & 36.05 \\
\hline Wood & 77.70 & 84.13 & 55.49 & 91.48 & 87.71 & 82.85 \\
\hline Alfalfa & 96.41 & 91.34 & 100 & 49.48 & 95.51 & 95.51 \\
\hline Oatas & 98.38 & 90.68 & 99.53 & 90.95 & 60.19 & 24.42 \\
\hline
\end{tabular}

Table 8. The comparison users and producers accuracy for MNF images (120 bands)

Table (8) shows the results of classification by overall accuracy parameter for pixel-based, SAM and MLC, and subpixel based (LSU) methods. Finally, comparison of overall accuracy is applied between the three classification methods. Table (9) shows this comparison.

\begin{tabular}{|c|c|c|c|c|c|c|}
\hline Method & \multicolumn{2}{|c|}{ LSU } & \multicolumn{2}{c|}{ MLC } & \multicolumn{2}{c|}{ SAM } \\
\hline Image & Original & MNF & Original & MNF & Original & MNF \\
\hline $\begin{array}{c}\text { Over. } \\
\text { Accuracy } \\
(\%)\end{array}$ & 86.07 & 89.05 & 79.54 & 81.04 & 44.84 & 74.86 \\
\hline
\end{tabular}

Table 9. The Comparison of overall accuracy (OA) (120 bands)

As can be seen form above Tables, the minimum difference the between pixels based accuracy and subpixel based accuracy which was performed on the MNF images are $8 \%$ and $12 \%$, respectively because pixel-based methods has high accuracy in 120 bands. It is clear that LSU is a robust procedure for hyperspectral images classification.

\section{6) Conclusion}

It can be concluded that: In all of the classification algorithms were used in this research, increasing the number of bands, makes increasing the classification accuracy consequently. Therefore, by development of remote sensing technology and using of the new sensors with hyperspectral capabilities in RS science, it is better to use these images in classification process. In pixel based classification approaches such as maximum likelihood classification and Spectral angle mapper, increasing the number of bands (above 120), decreases the accuracy of classification results. Therefore using new image processing tools and techniques are introduced for hyperspectral images classification is offered. By applying Minimum Noise Fraction (MNF) transformation on the hyperspectral images, the correlation and noises from bands can be removed and we can sort them according to their variance. In the bands with a high variance, the features can distinguish from each other in a better mode, therefore classification accuracy increased sensibly. Using MNF transformation on images which used for classification, the individual class's accuracy is increased about 15 to 20 percent from pixel based procedures and 9 percent from unmixing method, when applying on source image. Applying MNF transformation on the hyperspectral images reduces the dimensionality of hyperspectral image data for next processing. In pixel-based classification approaches such as maximum likelihood classification and spectral angle mapper, increasing the number of bands (more than 120)we have a decrease in accuracy of the classification results. But in linear unmixing method there is no limitation in the number of bands and by increasing the number of bands the remaining amount of spectral decomposition for each pixel is minimized i.e., accuracy of subpixel classification is increased. Table (10) shows that increasing the number of bands; decreasing the RMS error of each pixel in model. This decreasing of RMS error is obviously seen in both of the original and MNF images.

\begin{tabular}{|c|c|c|c|}
\hline \multicolumn{2}{|c|}{ Type of Images } & \multicolumn{2}{c|}{ LSU } \\
\cline { 3 - 4 } & org & mnf \\
\hline N.B & \multicolumn{3}{|c|}{} \\
\hline 20 & RMS Mean Error & 1.992245 & 0.118545 \\
\hline 50 & RMS Mean Error & 2.647923 & 0.128302 \\
\hline 87 & RMS Mean Error & 2.45367 & 0.128193 \\
\hline 120 & RMS Mean Error & 2.340894 & 0.126726 \\
\hline 155 & RMS Mean Error & 2.133099 & 0.124806 \\
\hline 175 & RMS Mean Error & 2.031512 & 0.123393 \\
\hline 195 & RMS Mean Error & 1.938037 & 0.122026 \\
\hline \multicolumn{4}{|c}{} \\
\hline $\begin{array}{c}\text { Average RMS Mean } \\
\text { Error }\end{array}$ & $\mathbf{2 . 2 1 9 6 2 6}$ & $\mathbf{0 . 1 2 4 5 7}$ \\
\hline
\end{tabular}

Table 10. RMS Error Mean unmixing

The comparison accuracy of subpixel and pixel-based classification is performed in several cases; including users and 
producers' accuracy for original images, users and producers' accuracy for MNF images and overall accuracy. Average of the comparison parameters is shown in the Table (11).

\begin{tabular}{|c|c|c|c|c|c|c|c|}
\hline Method & \multicolumn{2}{|c|}{ LSU } & \multicolumn{2}{|c|}{ MLC } & \multicolumn{3}{|c|}{ SAM } \\
\hline $\begin{array}{l}\text { Type of } \\
\text { images }\end{array}$ & Original & $\mathrm{MNF}$ & Original & MNF & Orig & & MNF \\
\hline $\begin{array}{c}\text { User. } \\
\text { Accuracy }(\%)\end{array}$ & 86.38 & 87.83 & 66.21 & 79.18 & 54. & & 69.13 \\
\hline $\begin{array}{c}\text { Prod. } \\
\text { Accuracy }(\%)\end{array}$ & 77.64 & 86.82 & 55.33 & 75.30 & 46. & & 66.56 \\
\hline $\begin{array}{c}\text { Over. } \\
\text { Accuracy }(\%)\end{array}$ & 86.07 & 89.05 & 79.54 & 81.04 & 44. & & 74.86 \\
\hline \multicolumn{8}{|c|}{ Comparison } \\
\hline Methods & \multicolumn{3}{|c|}{ SAM and $\angle S U$} & \multicolumn{4}{|c|}{$M L C$ and $\angle S U$} \\
\hline $\begin{array}{l}\text { Type of } \\
\text { images }\end{array}$ & \multicolumn{2}{|c|}{ Original } & MNF & \multicolumn{2}{|c|}{ Original } & & $\mathrm{INF}$ \\
\hline $\begin{array}{l}\text { Min.Diff. } \\
\text { User. A (\%) }\end{array}$ & \multicolumn{2}{|l|}{31.45} & 18.70 & \multicolumn{2}{|c|}{20.17} & & 65 \\
\hline $\begin{array}{c}\text { Min.Diff.Prod } \\
. \mathrm{A}(\%)\end{array}$ & \multicolumn{2}{|c|}{31.51} & 20.26 & \multicolumn{2}{|c|}{22.31} & & 1.52 \\
\hline $\begin{array}{l}\text { Min. Diff. } \\
\text { OA }(\%)\end{array}$ & \multicolumn{2}{|c|}{41.23} & 14.19 & \multicolumn{2}{|c|}{6.53} & & .01 \\
\hline
\end{tabular}

Table 11. The comparison of average accuracy for pixel based and subpixel classification (120 bands)

The table above reveals that, the spectral unmixing method that implemented here is an effective technique for classifying a hyperspectral image. Classification results by applies on the original images are not appropriate because some of the hyperspectral image bands are subject to absorption and they contain only little signal but more noise content. Also, applying the minimum noise fraction transformation on the hyperspectral images increased the individual classes accuracy of pixel based classification methods as well as unmixing method about 15 to 20 percent and 8 percent respectively. Therefore, linear spectral unmixing is in the beginning of its growth and will be found many applications in many of branch science in the future.

\section{7) References}

[1] Abkar A.A. (1999). "Likelihood-Based Segmentation and Classification of Remotely

Sensed". ITC publication number 73.

[2] Proffesor Maria Petrou "Tutorial on modern techniques in remote sensing", School of

Electronic Engineering, Information Technology and

Mathematics, University of Surrey, Gui

ldford,

httpllwww.survey.ntua.gr/main/Labs/rsens/DeCETI/

[3] P. Gong, J.R. Miller, J. Freemantle, and B. Chen. (1991).

"Spectral decomposition of Landsat

Thematic Mapper data for urban land-cover mapping", Calgary, Alberta, Canada.

[4] Dr. Robert A. Schowengerdt, (2000).,"Subpixel

Classification". http//:www.arizona.edu.

[5] J.J. Settle and N.A. Drake, (1993), "Linear mixing and the estimation of ground cover

proportions". International Journal of Remote Sensing.

[6] D. Heinz, C-I. Chang, M.L.G. Althouse,( 1999). "Fully constrained least-squares based linear

unmixing," International Geoscience and Remote Sensing Symposium. 